\title{
Spatially and temporally resolved measurements of the temperature inside droplets impinging on a hot solid surface
}

\author{
William Chaze ${ }^{1,2} \cdot$ Ophélie Caballina $^{1,2} \cdot$ Guillaume Castanet $^{1,2}$ (1) Fabrice Lemoine $^{1,2}$
}

Received: 16 March 2017 / Revised: 10 May 2017 / Accepted: 18 May 2017

(c) Springer-Verlag GmbH Germany 2017

\begin{abstract}
Heat transfers at the impact of a droplet on a hot solid surface are investigated experimentally. Millimetersized water droplets impinge a flat sapphire window heated at $600{ }^{\circ} \mathrm{C}$. The time evolution of the droplet temperature is characterized using the two-color laser-induced fluorescence technique. For that, a Q-switched Nd:YAG laser is used for the excitation of the fluorescence to obtain instantaneous images of the droplet temperature. Water is seeded with two fluorescent dyes, one sensitive to temperature (fluorescein disodium) and the other not (sulforhodamine 640). Owing to a wavelength shift between the dyes' emissions, the fluorescence signal of the dyes can be detected separately by two cameras. The liquid temperature is determined with a good accuracy by doing the ratio of the images of the dyes' fluorescence. A critical feature of the method is that the image ratio is not disturbed by the deformation of the impacting droplet, which affects the signals of the dyes almost identically. Experiments are performed in the conditions of film boiling. A thin vapor film at the interface between the droplet and the solid surface prevents the deposition of liquid on the hot solid surface. Measurements highlight some differences in the rate of heat transfers and in the temperature distribution within the droplet between the bouncing and splashing regimes of impact.
\end{abstract}

Guillaume Castanet

guillaume.castanet@univ-lorraine.fr

1 Université de Lorraine, LEMTA, UMR 7563, 54500 Vandœuvre-Lès-Nancy, France

2 CNRS, LEMTA, UMR 7563, 54500 Vandœuvre-Lès-Nancy, France

\section{Introduction}

Many industrial applications require a rapid cooling from high temperatures. In that purpose, the use of an evaporative liquid is almost unavoidable as it allows to take advantage of the latent heat of vaporization to dissipate higher heat fluxes. Liquid cooling includes pool boiling, impingement of liquid jets or sprays. Of the later technologies, spray cooling is certainly the most attractive for the thermal management of high heat flux systems (Labergue et al. 2015). Compared to jet impingement, it has the capability of cooling a relatively wider surface area with a single nozzle. It also has an unrivalled cooling efficiency, meaning that significant quantities of liquid coolant can be saved to remove the same amount of heat. These features explain why spray cooling has played an important role in several industrial applications, especially in metal production and processing industry. However, while this cooling technology is applied for decades, its integration remains a complex and cumbersome process because of still incomplete knowledge of the fluid flow and heat transfer characteristics. In particular, scientific investigations focused on individual droplets are still required to understand the underlying physics behind the interactions between droplets and a hot solid surface.

When a drop impacts a hot wall, different behaviors can be observed. The drop can spread over the solid surface and remain attached to it due to wettability forces. It can splash and create several smaller secondary droplets or simply rebound. Extensive experimental investigations were carried out to characterize the role played by the different parameters influencing the behavior of an impacting droplet. Among them, some were related to the dynamics of the impacting droplets (velocity, diameter, etc.), the physical 
properties of the liquid (viscosity, surface tension, etc.), and the solid surface (temperature, roughness, thermal effusivity, etc.) (Liang and Mudawar 2017; Moreira et al. 2010). Descriptions of an impact (maximum spreading diameter, resident time, coefficient of restitution, etc.) were usually made on the basis of correlations based on dimensionless numbers characterizing the relative magnitude of the forces acting on the impacting droplet, i.e., Reynolds, Weber and Ohnesorge numbers. A significant progress has been made in the numerical simulation of the drop impact, with an increasing number of studies using level-set/volume-of-fluid methods to track the liquid interface (Eggers et al. 2010; Roisman et al. 2009). However, in most cases, simulations are limited to isothermal or moderately heated solid surfaces and no phase change.

Regarding the heat transfers, almost all the experimental studies were focused around the heat removal from the solid surface, whose temperature was monitored using either thermocouples embedded in the wall thickness (Bernardin and Mudawar 1997) or IR thermography (Jung et al. 2016; Dunand et al. 2013; Labergue et al. 2017). Coupled with an inverse model, these measurements made it possible to quantify the heat removed from the wall and the cooling efficiency (Dunand et al. 2013; Labergue et al. 2017). However, the contributions of liquid evaporation and liquid heating (i.e., the latent heat and the sensible heat) to the overall heat exchange between the droplet and the wall are not obvious to evaluate when the wall heat flux is the only measured parameter. No information on the droplet heating was available in the literature until the pioneering work of Castanet et al. (2009). The latter made use of the two-color laser-induced fluorescence thermometry (2cLIF) which is one of the few proven techniques to characterize the temperature of droplets (Lemoine and Castanet 2013). The liquid (in this case water) is seeded by a temperaturedependent fluorescent dye. A stream of mono-sized d roplets impinging obliquely on a heated wall passes through a laser beam and the fluorescence emitted by the droplets is detected by photomultiplier tubes. The excitation volume of the fluorescence, whose dimensions are of the order of a few hundreds of microns, is moved from one side of the impact region to the other to characterize the temperature of the incoming and outgoing droplets. Subsequently, the technique was extended to polydisperse sprays (Labergue et al. 2017). Joint size/velocity/temperature distributions can be obtained by combining temperature measurements with Phase Doppler Analysis (PDA). For that, the fluorescence excitation was achieved by means of the two intersecting laser beams forming the probe volume of the PDA technique. Dunand et al. (2012) made use of a laser sheet and two cameras to visualize the temperature of the impacting droplets. However, using a CW laser led to serious limitations. Collecting signal from thousands of droplets was required to improve the signal-to-noise ratio and accurately measure the temperature. In other words, it was not possible to obtain a detailed evolution of the temperature within the droplets during their impingement on the wall. Only the global heating of the droplets after leaving the hot solid surface could be extracted. Nevertheless, Dunand et al. (2013) were capable of comparing the droplet heating and the heat removed from the wall (characterized by IR thermometry). Using an energy balance approach, they also quantified the evaporated mass of liquid during an impact.

More recently, several improvements to the 2cLIF technique were made by Chaze et al. (2016) who did use of a nanosecond pulsed laser to achieve single shot measurements of the temperature in a turbulent liquid jet. Also, Chaze et al. (2016) paid attention to the loss in temperature sensitivity undergone by several fluorescent dyes in the saturated regime of excitation of the fluorescence, which is usually encountered using of pulsed lasers. This led them selecting fluorescein disodium which is not affected by the same adverse effect of losing temperature sensitivity at high laser irradiance. The present study relies on this previous work. It aims at characterizing the instantaneous temperature of droplets impinging on a hot solid surfaces. The focus is put on the film boiling regime, in which a thin vapor film at the interface between the droplet and the solid surface prevents the deposition of liquid on the wall surface. Ultimately, the experimental results will help understanding the coupling between the droplet deformation at the impact and the heat and mass transfers. They will also be useful benchmarks to validate detailed numerical simulations which are taking into account phase change and heat transfers (Rueda Villegas et al. 2017).

\section{Principle of laser-induced fluorescence thermometry}

\subsection{Temperature dependence of the fluorescence signal}

Many examples of temperature measurements in liquids that are based on 2cLIF can be found in the literature. Applications are related to single-phase liquid flows (Kim et al. 2003; Lemoine et al. 1999; Sakakibara and Adrian 1999, 2004; Bruchhausen et al. 2005; Chaze et al. 2016), mono-sized droplet streams (Castanet et al. 2009, 2011; Dunand et al. 2012; Lemoine and Castanet 2013; Perrin et al. 2015) and sprays (Labergue et al. 2013; Lemoine and Castanet 2013). In most of these applications, a CW laser was utilized to induce fluorescence. However, for the purpose of instantaneous imaging, it is very advantageous, and even sometimes necessary to use pulsed laser sources, which allow delivering a considerable amount of energy over a very short period of time (typically a few ns 
with Q-switched Nd:YAG lasers). According to Chaze et al. (2016), the fluorescence $\mathrm{d} F_{\lambda}$ signal emitted at a wavelength $\lambda$ in an elementary volume $\mathrm{d} V$ of liquid can be expressed by

$\mathrm{d} F_{\lambda}=\eta \frac{\Omega}{4 \pi} \varepsilon_{0} \phi_{\lambda} \frac{I_{0}}{\left(1+I_{0} / I_{\text {sat }}\right)} C \mathrm{~d} V$.

In this expression, $I_{0}$ corresponds to the laser intensity in the volume $\mathrm{d} V . C$ is the molar concentration of the dye molecules. $\eta$ is the transmission efficiency of the fluorescence light to the detector. $\Omega$ denotes the solid angle of the collection. $\varepsilon_{0}$ is the molar extinction coefficient of the fluorescent molecules at the excitation wavelength of the laser beam. $\phi_{\lambda}$ stands for the fluorescence quantum yield. $I_{\text {sat }}$ is the saturation intensity of the fluorescent dye [typically a few MW/ $\mathrm{cm}^{2}$ according to Crimaldi (2008); Chaze et al. (2016)]. Several parameters in Eq. (1) are temperature dependent, $\varepsilon_{0}, \phi_{\lambda}$ and $I_{\text {sat }}$ being the most important. While $\phi_{\lambda}$ decreases with the temperature due to collisional quenching, the saturation intensity $I_{\text {sat }}$ follows an inverse trend (Chaze et al. 2016). As a result, a fluorescent dye can lose some of its temperature sensitivity at high laser irradiance. This effect was demonstrated experimentally by Chaze et al. (2016) who observed a significant decrease in the temperature response of rhodamine $\mathrm{B}$ and kiton red for an excitation energy such as $I_{0} \gg I_{\text {sat }}$. In the case of these dyes, the temperature sensitivity of the fluorescence signal mainly arises from the variation of the fluorescence quantum yield. In contrast, other dyes, such as fluorescein disodium (renamed FL in the following) retains a high and unchanged temperature sensitivity regardless of the laser irradiance. For this reason, FL is chosen in the following to perform single shot measurements of the temperature.

\subsection{Fluorescence properties of fluorescein disodium}

A preliminary study was carried out to characterize the fluorescence of FL in the outlook of droplet measurements. A transmission spectrometer was used first to measure the absorption coefficient of FL at different wavelengths (Fig. 1). Despite a rather low absorption at $532 \mathrm{~nm}$, a frequency-doubled Nd:YAG laser will be used in the following to induce the fluorescence of FL. It has already been demonstrated that an excitation at this wavelength is well adapted for temperature measurements (Sutton et al. 2008; Chaze et al. 2016). The effect of the temperature on the fluorescence signal was investigated using a laser beam at $532 \mathrm{~nm}$ which passes through a glass cuvette holding the fluorescent dye in solution into water. As the fluorescence of FL is very sensitive to $\mathrm{pH}$ (Coppeta and Rogers 1998), $\mathrm{pH}$ is stabilized at 5.6 using a phosphate buffer. The liquid inside the cuvette is progressively heated while a stirring is applied to insure a uniform temperature. The emitted fluorescence is collected onto a spectrometer. Two

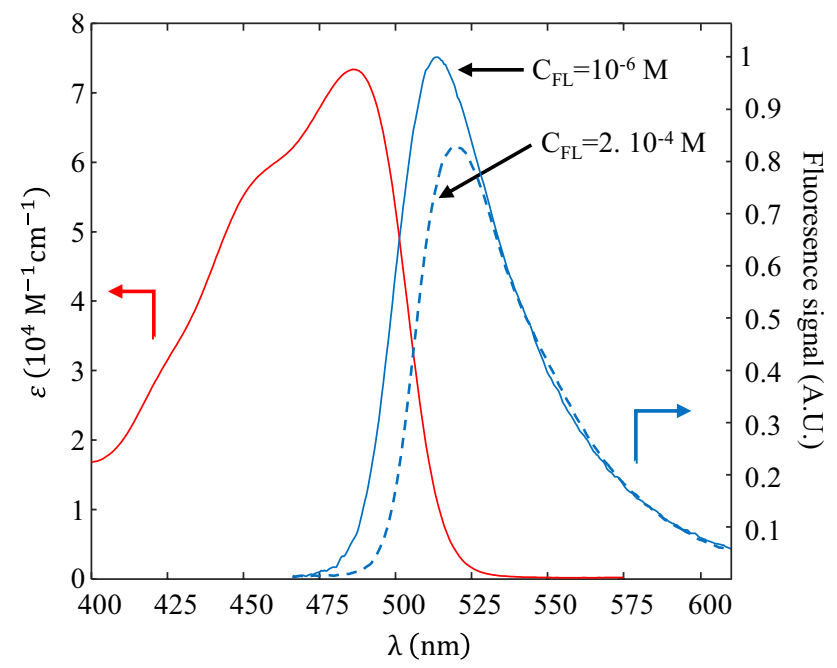

Fig. 1 Absorption and emission spectra of $\mathrm{FL}$ at $20{ }^{\circ} \mathrm{C}$ and $\mathrm{pH}=$ 5.6. The emission spectra are normalized, so that they are superimposed for the wavelengths above $540 \mathrm{~nm}$. The optical thickness for self-absorption is $2.5 \mathrm{~mm}$ in the measurements of the emission spectrum

concentrations of FL are considered in the preliminary tests, $C_{\mathrm{FL}}=10^{-6} \mathrm{M}$ and $C_{\mathrm{FL}}=2.10^{-4} \mathrm{M}$, the latter being reused in the study of the drop impacts.

As shown in Fig. 1, the emission spectra measured at the two concentrations appear to be shifted. This shift is due to self-absorption of the fluorescence, which is significant below $540 \mathrm{~nm}$ at $C_{\mathrm{FL}}=2.10^{-4} \mathrm{M}$ even if the optical thickness is limited to $2.5 \mathrm{~mm}$ (this length corresponds to the droplet size in Sects. 3 and 4). In practice, detecting the fluorescence signal below $540 \mathrm{~nm}$ is not recommended since the signal would depend on the droplet shape, given that the experiments will deal with millimeter-size drops. Moreover, the absorption cross-section of FL is temperature dependent, and thus self-absorption is likely to affect the temperature sensitivity of the signal in the bands which are reabsorbed (Coppeta and Rogers 1998; Chaze et al. 2016).

In Fig. 2, it can be seen that the recorded emission spectra increase significantly with the temperatures between 20 and $80{ }^{\circ} \mathrm{C}$. This variation of the fluorescence signal is compared with that of the coefficient absorption at the laser wavelength which was measured in parallel (Fig. 3). Two photodiodes are used to monitor the intensities of the incident and outgoing laser beam, and thus to determine the beam attenuation in the cuvette. In the range from 20 to $80^{\circ} \mathrm{C}$, the fluorescence signal and the absorption coefficient $\varepsilon_{0}$ at the laser wavelength follow a same trend (Fig. 3). Referring to Eq. (1), this demonstrates that the sensitivity to temperature of the fluorescence signal comes exclusively from the variation of $\varepsilon_{0}$. To perform this comparison, only 


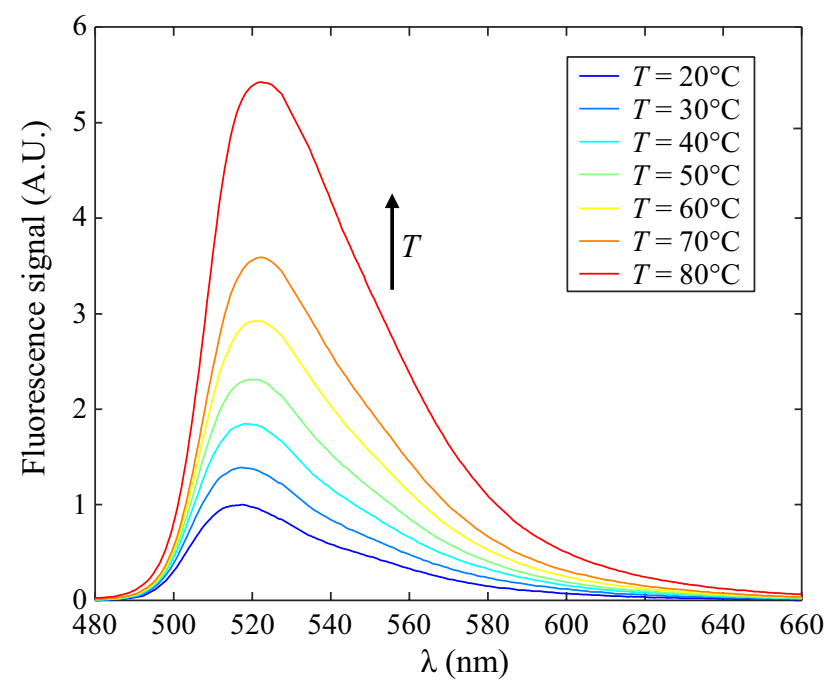

Fig. 2 Fluorescence emission spectrum of FL measured at various temperatures $\left(C_{\mathrm{FL}}=2.10^{-4} \mathrm{M}\right.$ and the optical thickness is $\left.2.5 \mathrm{~mm}\right)$

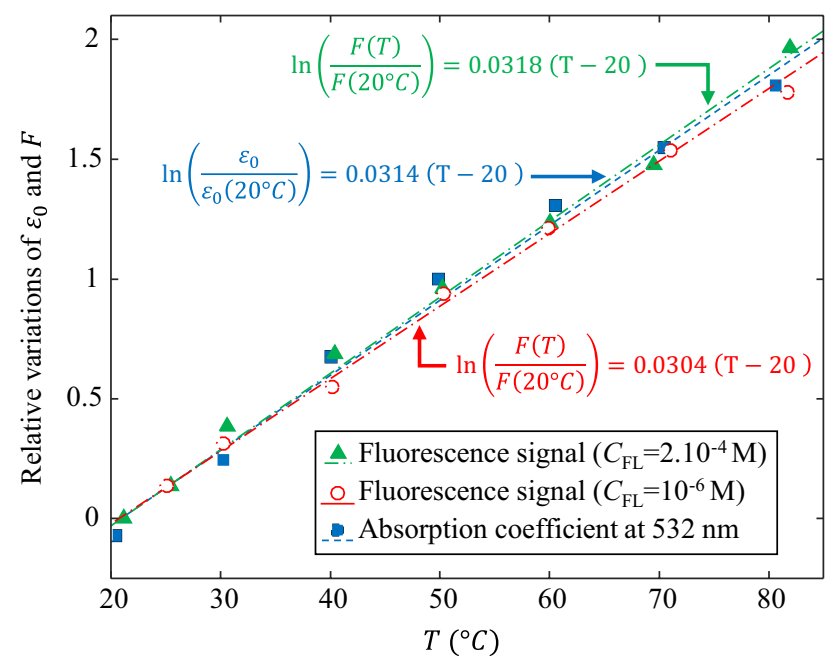

Fig. 3 Influence of the temperature on the fluorescence signal $F$ and the absorption coefficient $\varepsilon_{0}$ at $532 \mathrm{~nm}$

the wavelengths above $540 \mathrm{~nm}$ were considered in the fluorescence signal $F$ calculated from Fig. 2. This was required to eliminate much of the effects of self-absorption which are significant at $C_{\mathrm{FL}}=2.10^{-4} \mathrm{M}$ and for an optical path length of $2.5 \mathrm{~mm}$. Between 20 and $80^{\circ} \mathrm{C}$, the variations of the fluorescence signal $F$ and the absorption coefficient $\varepsilon_{0}$ can be described by

$\frac{x}{x_{0}}=\exp \left\{s\left(T-T_{0}\right)\right\}$

where $x=F$ or $\varepsilon_{0}$ and $x_{0}=x\left(T_{0}\right)$. The coefficient $s=\frac{1}{x} \mathrm{~d} x$ corresponds to the temperature sensitivity. It is very close to $3.1 \% /{ }^{\circ} \mathrm{C}$ for both the fluorescence signal and the absorption coefficient at $532 \mathrm{~nm}$. In Fig. 3, s appears to be slightly higher at $C_{\mathrm{FL}}=2.10^{-4} \mathrm{M}$ than at $C_{\mathrm{FL}}=10^{-6} \mathrm{M}$. This difference is small $\left(3.18 \% /{ }^{\circ} \mathrm{C}\right.$ against $\left.3.04 \% /{ }^{\circ} \mathrm{C}\right)$ but it cannot be totally explained by measurement errors. It certainly results from the re-induced fluorescence, i.e., the fluorescence induced by self-absorption. In the case $C_{\mathrm{FL}}=2.10^{-4}$ $\mathrm{M}$, re-induced fluorescence was estimated from Fig. 1 to account for about $30 \%$ of the overall fluorescent signal. Some of the re-induced fluorescence is expected to be more sensitive to temperature given that it is induced by two or more successive absorptions and that absorption is thermally dependent.

The value of $+3.1 \% /{ }^{\circ} \mathrm{C}$ for the coefficient $s$ is close to that of $+3.22 \% /{ }^{\circ} \mathrm{C}$ reported by Chaze et al. (2016). Coppeta and Rogers (1998) pointed out an effect of the temperature on the absorption coefficient of FL. They observed that the absorption coefficient increases with the temperature for $\lambda>495 \mathrm{~nm}$ while it decreases for $\lambda<495 \mathrm{~nm}$. This certainly explains the differences in temperature sensitivities $s$ in the literature for FL. The coefficient $s$ was evaluated at $-0.3 \% /{ }^{\circ} \mathrm{C}$ by Walker (1987) at $\lambda_{\mathrm{ex}}=488 \mathrm{~nm}$, while $+2.48 \% /{ }^{\circ} \mathrm{C}$ was obtained at $\lambda_{\mathrm{ex}}=514.5 \mathrm{~nm}$ by Coppeta and Rogers (1998). The value of $s$ in the present study also compares with that of fluorescein 27 (also named Fluorescein 548) which has a sensitivity of $+3.5 \% /{ }^{\circ} \mathrm{C}$ at $\lambda_{\mathrm{ex}}=532 \mathrm{~nm}$ according to Sutton et al. (2008). The latter also found for fluorescein 27 that the temperature variation of the fluorescence signal compares with that of $\varepsilon_{0}$ at 532 $\mathrm{nm}$. In this study, the disodium salt of fluorescein (FL) was preferred as it is very soluble in water. Also, Chaze et al. (2016) have verified the temperature response of FL is not affected in the saturated regime of excitation.

\subsection{Temperature measurements based on two-color laser-induced fluorescence}

At the droplet surface, light is refracted and reflected. As a consequence of light interaction with the droplet, the laser intensity $I_{0}$ and the transmission coefficient $\eta$ in Eq. 1 vary continuously when a droplet undergoes a deformation of its shape. The ratio of the signals detected in two spectral bands allows eliminating the dependence on the droplet shape, provided that these two bands are affected in an identical manner by light scattering and absorption. The detection bands must also have temperature sensitivities as different as possible for their ratio to vary sufficiently with temperature. A first solution is the use of a single dye (for example, rhodamine B, kiton red, pyrromethene 597-8C9), whose emission spectrum deforms with temperature (Lemoine and Castanet 2013). Another solution is to mix two dyes having a different temperature sensitivity and an emission shifted in wavelength (Sakakibara et al. 1993). This second approach was retained since the 
emission spectrum of FL does not deform with temperature (Chaze et al. 2016). FL is associated with sulforhodamine 640 (noted hereafter SR640) in a similar way than Chaze et al. (2016). The fluorescence signal of SR640 does not vary with temperature regardless the detection wavelength and the laser irradiance. FL and SR640 are heavy molecules which do not evaporate. Therefore, a change of their relative concentrations can be safely ignored in evaporative conditions. Figure 4 shows the detection bands selected for the experiments

- Band 1 [540-560 nm]: Only FL has a contribution in this band. Fluorescence is partially absorbed by SR640 $\left(\varepsilon \approx 80,000 \mathrm{M}^{-1} \mathrm{~cm}^{-1}\right)$.

- Band $2[>640 \mathrm{~nm}]$ : The emission of SR640 is predominant. However, the emission of FL is not totally negligible and its contribution depends on the respective concentrations of the dyes.

Neglecting the absorption by SR640 and the emission of FL in band 2, the ratio $R$ of the signals in the two detection bands can be determined from Eq. (1)

$R=\frac{F_{1}}{F_{2}} \propto \frac{\varepsilon_{0, F L}}{\varepsilon_{0, \mathrm{SR} 640}} \frac{C_{\mathrm{FL}}}{C_{\mathrm{SR} 640}} R_{\mathrm{sat}}$

where $R_{\mathrm{sat}}=\frac{1+I_{0} / I_{\mathrm{sat}} \mathrm{SR} 640}{1+I_{0} / I_{\mathrm{sat}, \mathrm{FL}}}$. Experiments will be carried out in the saturated regime of excitation (i.e., $I_{0} \gg I_{\text {sat }}$ ), meaning that $R_{\text {sat }} \approx 1$. Furthermore, Chaze et al. (2016) verified experimentally that $R$ does not vary significantly with $I_{0}$ including in the partially saturated domain of excitation $\left(I_{0} \approx I_{\text {sat }}\right)$. It is, therefore, expected that

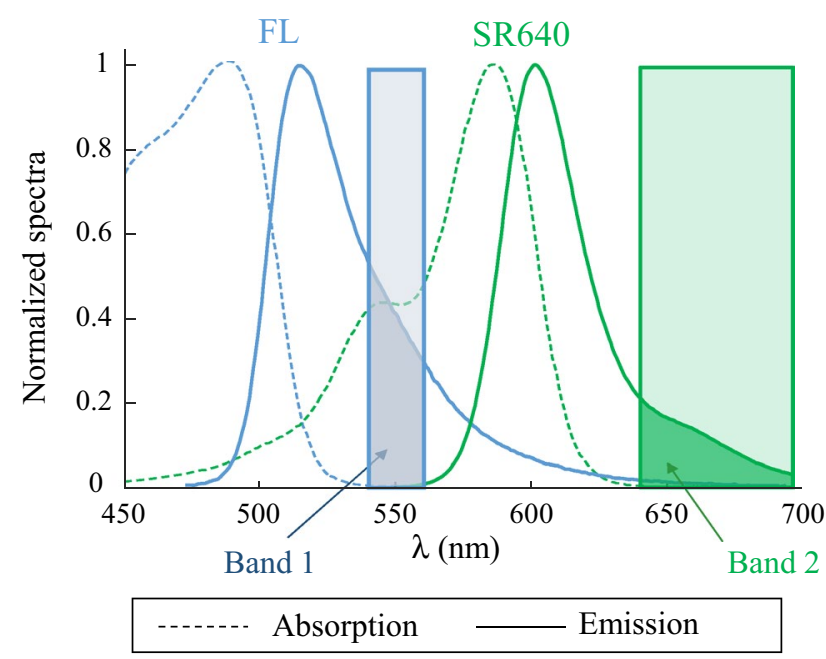

Fig. 4 The two detection bands selected for the application of the 2cLIF technique using the couple of dyes FL/SR640
$R=R_{0} \frac{\varepsilon_{0, \mathrm{FL}}(T)}{\varepsilon_{0, \mathrm{FL}}\left(T_{0}\right)}$

where $R_{0}$ is the reference ratio measured at a known temperature $T_{0}$.

\section{Application to the measurement of the temperature of impacting drops}

\subsection{Experimental setup}

The experimental setup is presented in Fig. 5. Millimeter-size drops are generated in a very reproducible manner by a syringe. When the drop pending to the tip of the needle exceeds a certain size, it detaches itself. A $400 \mu$ $\mathrm{m}$ base diameter needle is used to produce water drops with a diameter of $2.5 \mathrm{~mm}$. This diameter typically varies by less than $0.3 \%$ from one droplet to another. The frequency of the droplet detachment is controlled by means of a syringe drivers which allows delivering a constant liquid flow rate. The free-falling droplets impinge a sapphire window (2.54 cm in diameter and $5 \mathrm{~mm}$ thick) placed on a steel holder which is heated at $600{ }^{\circ} \mathrm{C}$ using cartridge heaters $(4 \times 250 \mathrm{~W})$. This temperature largely exceeds the static Leidenfrost temperature (estimated at $250{ }^{\circ} \mathrm{C}$ ) to avoid any wetting of the solid surface whatever the droplet velocity. Owing to the high thermal conductivity of sapphire $\left[12 \mathrm{~W} / \mathrm{m} \mathrm{K}\right.$ at $600{ }^{\circ} \mathrm{C}$ according to

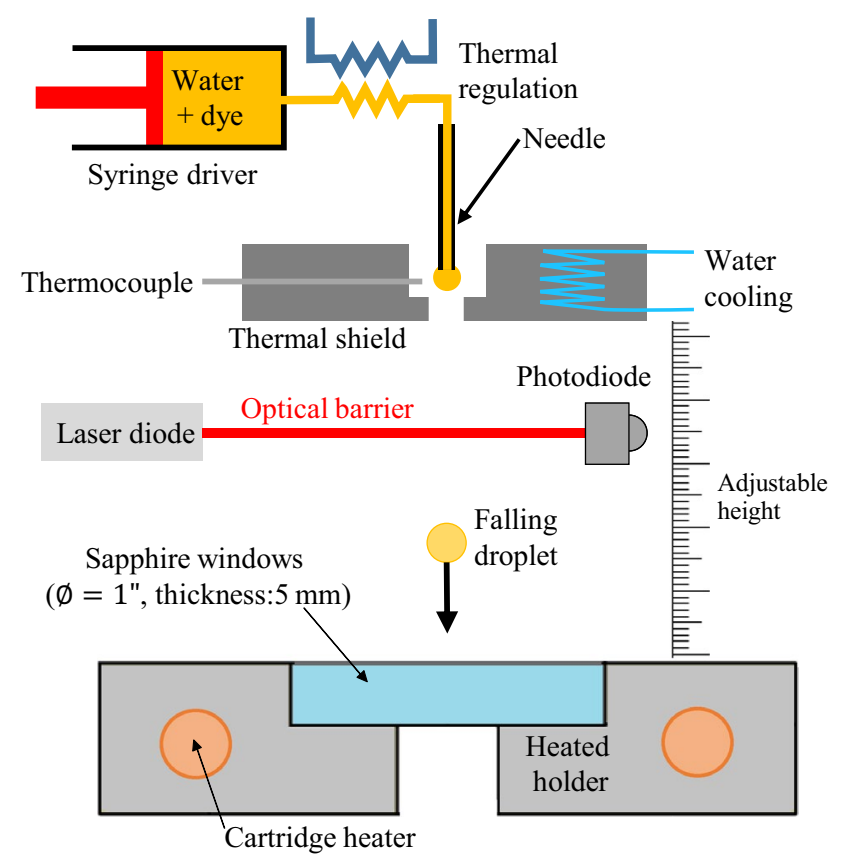

Fig. 5 The experimental setup 
Dobrovinskaya et al. (2009)], the temperature of the solid surface is uniform and almost equal to that of the steel holder. A thermal shield is necessary to prevent the heating of the liquid inside the needle given the hot air plume rising from the sapphire window. The tip of the needle is placed into a cavity of a few millimeters arranged inside a metallic plate which is cooled down by a water circulation. Moreover, the fluorescent solution passes into a heat exchanger before entering the needle. A thermocouple, placed at a short distance of the droplet in the cavity, is used to control the ambient temperature. This provides a good approximation for the temperature of the pendant droplet, because the same setpoint is imposed in the thermal regulations with the heat exchanger and the thermal shield. The needle is moved up and down to modify the impact velocity. Finally, an aperture managed in the steel holder allows using a sapphire window for a backside illumination and visualization of the impacting droplet.

A pulsed Nd:YAG laser at $532 \mathrm{~nm}$ (Quantel Brillant B, pulse energy $E_{\max }=450 \mathrm{~mJ}$, pulse duration $\mathrm{d} t=5 \mathrm{~ns}$ ) is utilized for the excitation of the fluorescence. The laser beam is much larger than the droplets, which yields an illumination of the whole liquid volume during the droplet deformation. Experiments are carried out at high laser fluence (typically $I_{0}>100 \mathrm{MW} / \mathrm{cm}^{2}$ ), so that the fluorescence is saturated at each laser shot. Hence, the fluorescence emission is distributed quite uniformly inside the droplets. The repetition rate of the pulsed laser $(10 \mathrm{~Hz})$ is by far too low to resolve temporally a drop impact. However, a time reconstruction is possible owing to the fact that droplets can be produced repeatedly with exactly the same size, velocity and trajectory when all aerodynamic perturbations are eliminated. An optical barrier consisting of a laser diode and a photodiode, placed a few millimeters above the sapphire surface, makes it possible to detect the fall of a droplet. The time delay between the detection by the barrier and the triggering of the laser pulse is varied by small increments so as to explore, droplet after droplet, the whole period of an impact. As displayed in Fig. 6, droplets are observed by means of two CCD cameras (Allied Vision Tech Prosilica GT3300 B/C GigE Camera $3296 \times 2472,12$ bits, $5.5 \mu \mathrm{m}$ ) each one equipped with an interference filter for the detection of the fluorescence in the bands presented in Fig. 4. The optical system also includes an objective lens (SIGMA APO MACRO $150 \mathrm{~mm}$ F2.8 EX DG OS HSM and its teleconverter $\times 2$ ) and a beamsplitter mounted in front of the cameras. A high pass filter $(\lambda>542 \mathrm{~nm})$ is added between the objective lens and the beamsplitter to eliminate more efficiently the scattered laser light. A pellicle beamsplitter (thickness $2 \mu \mathrm{m}$ ) is selected to avoid having ghost images from second surface reflections. Its reflection/transmission

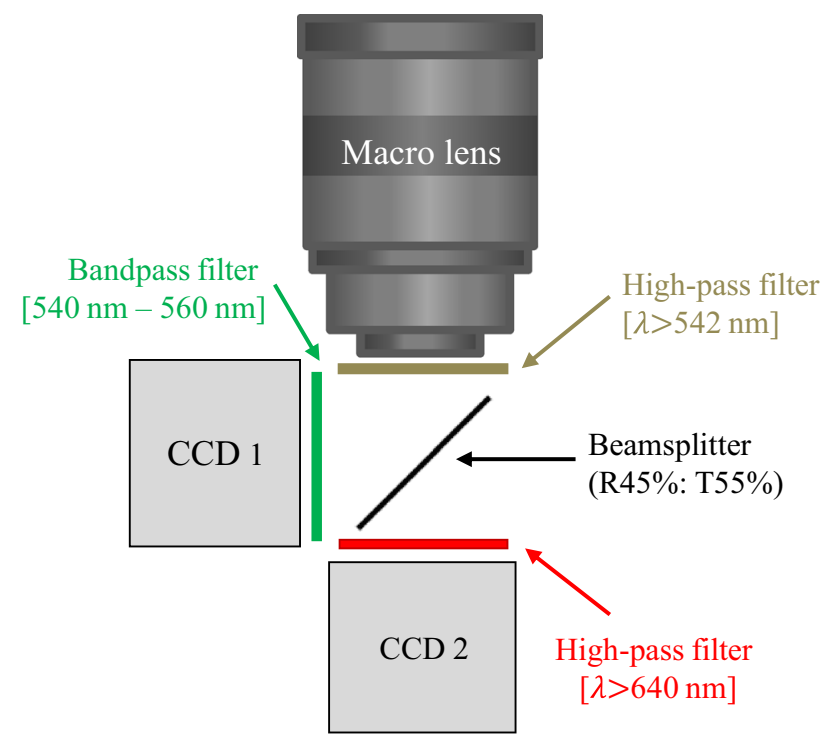

Fig. 6 Arrangement of the optical detection system

ratio is very weakly wavelength-dependent. Two configurations of the optical system are used to visualize the impacting droplet (Fig. 7). A $45^{\circ}$ tilted mirror is placed under the sapphire window and its heated holder. It makes it possible to observe the droplet from below with the cameras, while a side illumination by the laser is performed (Fig. 7a). The same mirror can also be used to illuminate the droplet from below, while the cameras are taking side view images of the droplet (Fig. 7b).

\subsection{Image processing and quantitative measurements}

To obtain quantitative measurements of the temperature, a specific processing of the images is necessary. The steps of this processing, already described by Dunand et al. (2012), include

- a correction for the possible non-linearity of the camera responses

- a correction for geometric and chromatic aberrations of the imaging system

- a repositioning of the pair of images given that the cameras may not have exactly the same field of view

- a conversion of the fluorescence ratio into temperature by means of a calibration and a reference measurement.

Corrections for the optical aberrations and the non-linearity of the detectors proved to be unnecessary with the present measurement system. 
Fig. 7 The two optical configurations used to visualize the fluorescence emission of the impinging droplet a bottom view imaging, b side view imaging
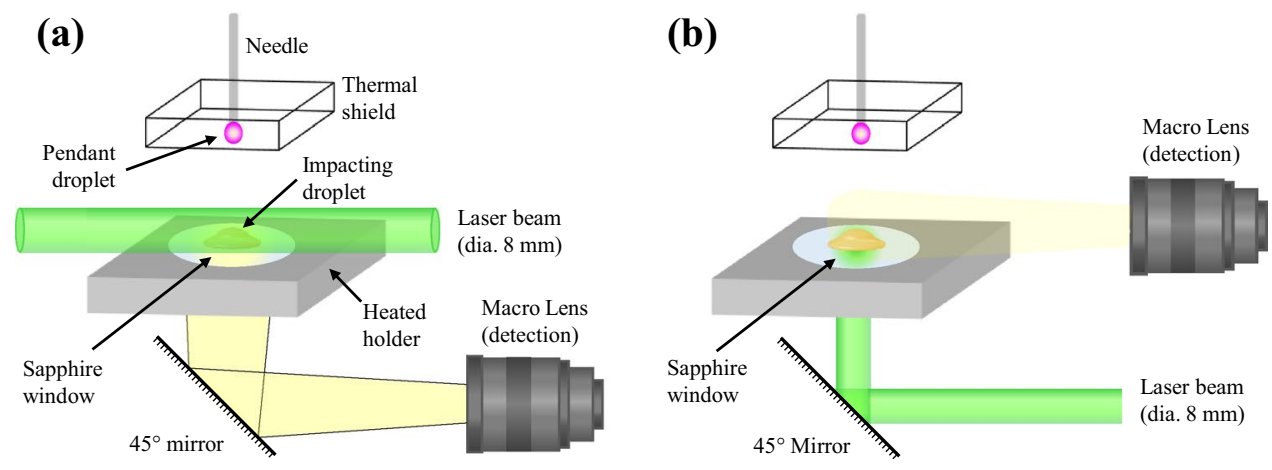

\subsubsection{Pixel correspondence between the cameras}

An offset of a few pixels between the images of the two cameras may remain despite a careful alignment of the beamsplitter. For this reason, a calibration of the image coordinates is performed following a procedure already described by Sakakibara and Adrian (1999). A regular grid of circular dots (diameter: $50 \mu \mathrm{m}$, spacing: $125 \mu \mathrm{m}$ ) is positioned to obtain focused images of the dots. Then, a recognition algorithm is used to find the coordinates of each dot in the images. A third order polynomial function is created to obtain a perfect matching between the coordinates of the dots in the images of the two cameras (the function $c p 2$ tform of MATLAB is used). The grid pattern allows to have at least 1500 control points in the image to determine the coefficient of the transform. This large number of control points insures an accurate positioning throughout the whole field of view. The residual error on the pixel positioning is estimated to be of the order of 10 $\mu \mathrm{m}$ in the worst case. This value is about equal to the spatial resolution of the side view images and two times less than the resolution of the bottom view images, which are presented in Sect. 4. Given that geometrical aberrations are almost not present in the optical system, the transform is used to correct for a misalignment (i.e., a rotation $\theta$ of the beamsplitter and a translation $\left(t_{x}, t_{y}\right)$ of a camera). So normally, only three parameters $\left(\theta, t_{x}, t_{y}\right)$ are enough for a good positioning of the pixels as shown by Dunand et al. (2012) using a rather similar optical system. Here, the third order polynomial function contains ten coefficients. Obviously, their values are not the same if the magnification and the working distance of the macro lens are modified. The third order polynomial transform is applied to the images before doing the ratio and determine the temperature.

\subsubsection{Temperature calibration and dye concentrations}

The fluorescence ratio $R$ calculated by dividing the images of the two cameras is converted into temperature using a calibration curve. Presently, the calibration was carried out in a glass cube cell of $200 \mathrm{~mL}$ in which the fluorescent solution is heated progressively from 20 to $80{ }^{\circ} \mathrm{C}$. Images are recorded in the two detection bands and the fluorescence ratio is determined after repositioning the pixels. The evolution of the fluorescence ratio $R$ is plotted as a function of the temperature in Fig. 8. In Fig. 8a, the calibration curves correspond to the dye mixture $C_{\mathrm{SR} 640}=0.710^{-6} \mathrm{M}$ and $C_{\mathrm{FL}}=2.10^{-4} \mathrm{M}$ (used later to investigate the drop impact). The temperature sensitivity of the fluorescence ratio can be evaluated at $2.7 \% /{ }^{\circ} \mathrm{C}$, which is slightly lower than expected using Eq. (4). The difference is due to the residual emission of FL in the
Fig. 8 Evolution of the fluorescence ratio $R$ as a function of the temperature. $R$ is normalized to be equal to 1 at $23{ }^{\circ} \mathrm{C}$. a $C_{\text {SR640 }}=7.10^{-7} \mathrm{M}$ and $C_{\mathrm{FL}}=2 \cdot 10^{-4} \mathrm{M}, \mathbf{b}$ $C_{\mathrm{FL}}=2 \cdot 10^{-4} \mathrm{M}$ (a)

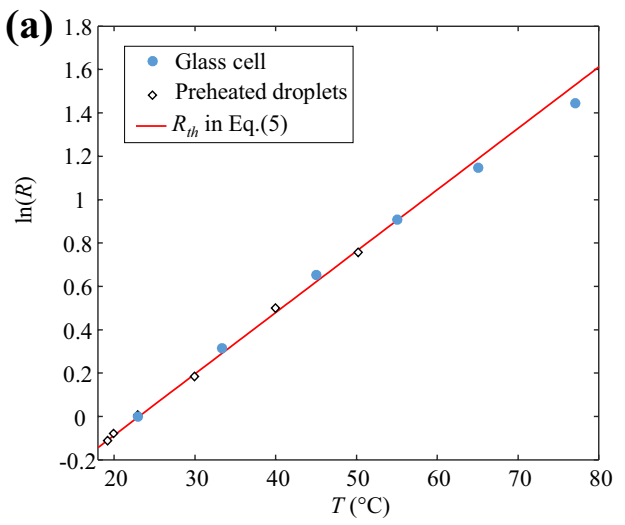

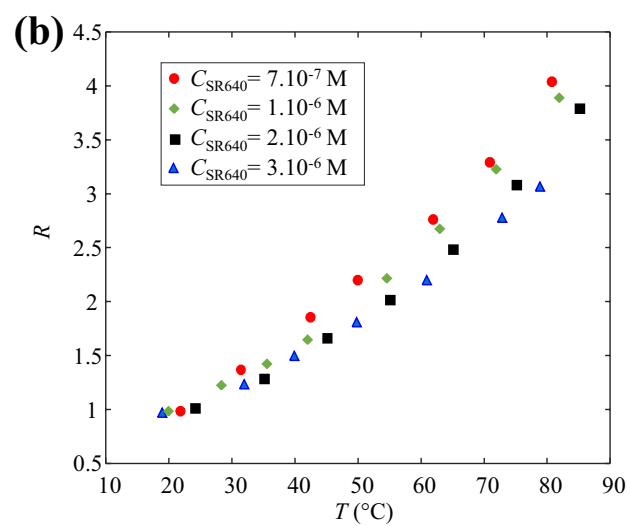


detection band of SR640. For these dye concentrations, further investigations revealed that the emission of FL accounts for $2.9 \%$ of the signal in the band $[\lambda>640 \mathrm{~nm}]$ at $20{ }^{\circ} \mathrm{C}$. Equation (4) can be corrected to take into account this residual contribution of FL:

$R_{\mathrm{th}}=\frac{F}{(1-0.029)+0.029 F}$

where $F=\exp (s(T-20))$ and $s=3.1 \% /{ }^{\circ} \mathrm{C}$. The evolution of $R_{t h}$ is plotted in Fig. 8 and a good agreement with the experiments can be observed. Only a small deviation can be pointed out above $60{ }^{\circ} \mathrm{C}$. SR640 undergoes an irreversible degradation when it is heated (Chaze et al. 2016). This degradation process is illustrated in Fig. 9. The fluorescence of SR640 decreases with time when it remains in a warm environment for several minutes. This phenomenon is not a problem for studying the impact of droplets (lasting a few ms), but it makes the calibration in temperature of the fluorescence ratio more difficult. Despite a rapid heating of the liquid in the calibration cell, a small part of SR640 was probably degraded during the calibration, which would explain the small deviation above $60{ }^{\circ} \mathrm{C}$ pointed out in Fig. 8. It should be also added that a gradual decrease in the signal of the SR640 was observed, when it is placed under a continuous illumination or repeated laser shots. This photodegradation process only occurs when FL and SR640 are present. It is not found when both dyes are alone in solution. A special care was, therefore, taken to limit the number of laser shots during the calibration and the liquid was also stirred in the cell. For validation of Eq. (5), droplets were also heated prior to their detachments from the needle, and the fluorescence ratio was evaluated in-flight from their images. Here also, a good agreement can be observed, but this test was limited to $50{ }^{\circ} \mathrm{C}$ due to the thermal degradation of SR640 in the liquid circuit prior to the droplet detachment.

It should be emphasized that a loss in temperature sensitivity occurs at high concentrations in dye molecules due to energy transfers between the two fluorescent species. These transfers can be induced by a radiative process. The emission of FL is absorbed by SR640 which emits some fluorescence in returns. Non-radiative transfers due to a dipole-dipole coupling mechanism, called Förster resonance energy transfer (FRET), are also possible (Lakowicz 2006). A condition for FRET to take place, is an overlap between the emission spectrum of the donor and the absorption spectrum of the acceptor. In the present case, FL can initiate a fluorescence of SR640 with the FRET mechanism. The efficiency of FRET is extremely sensitive to small changes in concentration when the distance between the donor (FL) and the acceptor (SR640) approaches the distance of Forster

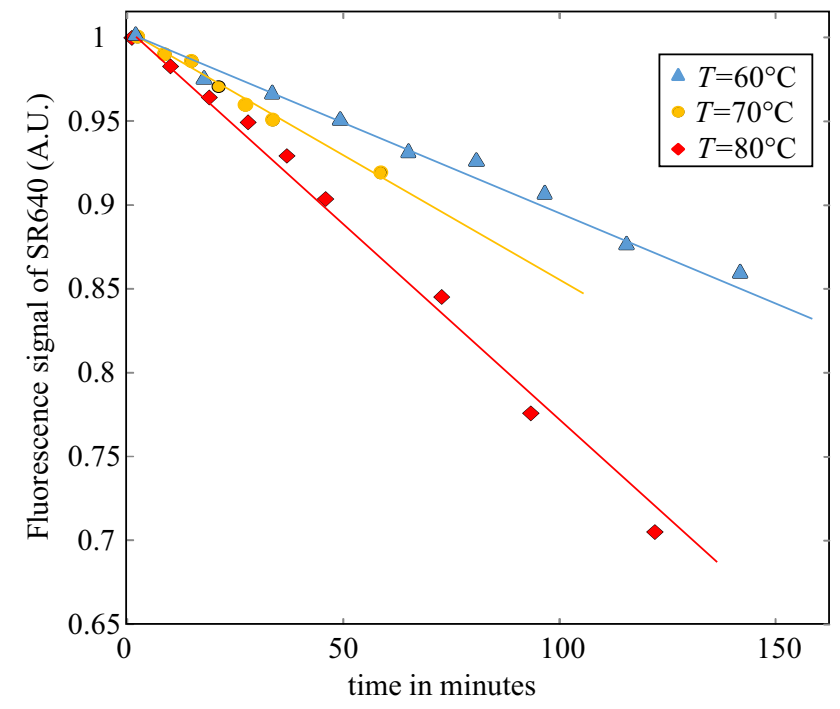

Fig. 9 Illustration of the thermal degradation of SR640. Evolution of the fluorescence signal of SR640 when it remains for several minutes at different temperatures

$r_{\mathrm{f}}$. The fraction of energy $e$ transferred by FRET can be expressed by

$e=\frac{1}{1+\left(r / r_{\mathrm{f}}\right)^{6}}$.

The radius $r_{\mathrm{f}}$ is typically on the order of a few $\mathrm{nm}$. In principle, $r_{\mathrm{f}}{ }^{6} \sim J$ where $J$ is the spectral overlap integral between the donor emission spectrum $f_{\mathrm{D}}(\lambda)$ and the acceptor absorption coefficient $\varepsilon_{\mathrm{A}}(\lambda)$ (Lakowicz 2006):

$J=\frac{\int f_{\mathrm{D}}(\lambda) \varepsilon_{A}(\lambda) \lambda^{4} \mathrm{~d} \lambda}{\int f_{\mathrm{D}}(\lambda) \mathrm{d} \lambda}$.

Energy transfers between excited forms of FL and SR640 are detrimental to the measurements. This can be illustrated by increasing the concentration of SR640 while maintaining the concentration of FL at $2 \cdot 10^{-4} \mathrm{M}$ (Fig. 8b). Although the temperature sensitivity of $R$ is expected to increase with $C_{\mathrm{SR} 640}$ (given that the contribution of FL in the detection band of SR640 decreases with increasing $C_{\mathrm{SR} 640}$, see the above discussion leading to Eq. 5), an inverse trend is observed. This effect is not the result of the fluorescence of SR640 induced by absorption of FL emission. Indeed, the temperature sensitivity of $R$ proved to be unaffected when the optical path is changed by several centimeters. Consequently, FRET is very likely in this case. Examples of FRET between FL and several types of rhodamines have been reported for dye concentrations of the same order as Fig. 8b [in particular, FRET in FL/ rhodamine $6 \mathrm{G}$ mixtures was investigated by Saha et al. (2015)]. 
As explained, the temperature sensitivity of the fluorescence ratio depends on the dyes' concentrations (Fig. 8b). Measurement error can occur if $C_{\mathrm{FL}}$ and $C_{\mathrm{SR} 640}$ increase during the experiments, as FRET is enhanced. Even though SR640 and FL do not evaporate with water, their concentrations are not expected to change significantly during a drop impact. Several experimental studies have shown that the cooling efficiency $E_{\text {cool }}=Q_{\mathrm{w}} /\left[m \cdot\left(L_{\mathrm{v}}+C p_{1}\left(T_{\text {sat }}-T_{0}\right)\right)\right]$ is limited to a few percents in the film boiling regime (Dunand et al. 2013; Jung et al. 2016), where $Q_{\mathrm{w}}$ is the heat removed from the solid wall by the drop, $T_{\text {sat }}$ is the liquid saturation temperature, $L_{\mathrm{v}}$ and $C p_{1}$ are the latent heat of vaporization and the heat capacity of liquid water. This means that it is not possible that the evaporation exceeds a few percent of the initial mass of the droplet, especially since a significant part of $Q_{\mathrm{w}}$ has also to be used to heat up the liquid prior to its evaporation.

\subsubsection{Reference measurement}

A reference point is needed to convert the fluorescence ratio into temperature. Denoting $f$ the calibration function (Fig. 8) and $R$ the measured value of the fluorescence ratio, the temperature is given by
$T=f^{-1}\left(\frac{R}{R_{0}} f\left(T_{0}\right)\right)$,

where $R_{0}$ and $T_{0}$ are, respectively, the fluorescence ratio and the temperature at the reference point. $R_{0}$ is determined from images in which the droplet has just impinged the sapphire surface (images referred at $t=0$ in Figs. $10,11,12,13,14) . T_{0}$ is assimilated to the temperature measured by the thermocouple inserted in the thermal shield (Fig. 5). It is assumed that the droplets do not heat up significantly when they travel in the hot air plume above the sapphire window. This can be justified by solving the heat equation for a droplet assimilated to a rigid sphere of mass $m$ and diameter $d$,

$m C p \frac{\mathrm{d} T}{\mathrm{~d} t}=\frac{N u \lambda_{g}}{d}\left(T_{\mathrm{amb}}-T\right)$,

where the Nusselt number $N u$ can be evaluated using $N u=2+0.6 \operatorname{Re}^{0.5} \operatorname{Pr}^{0.33}$ (Ranz and Marshall 1952). Equation (9) yields a drop heating of $0.3{ }^{\circ} \mathrm{C}$ in the case of a falling distance of $20 \mathrm{~cm}$ (corresponding to $W e=140)$. The heating of the droplet by the thermal radiation of the solid wall at $600{ }^{\circ} \mathrm{C}$ is even more negligible.
Fig. 10 Side and bottom views of the temperature field within an impacting droplet at $W e=10.2$

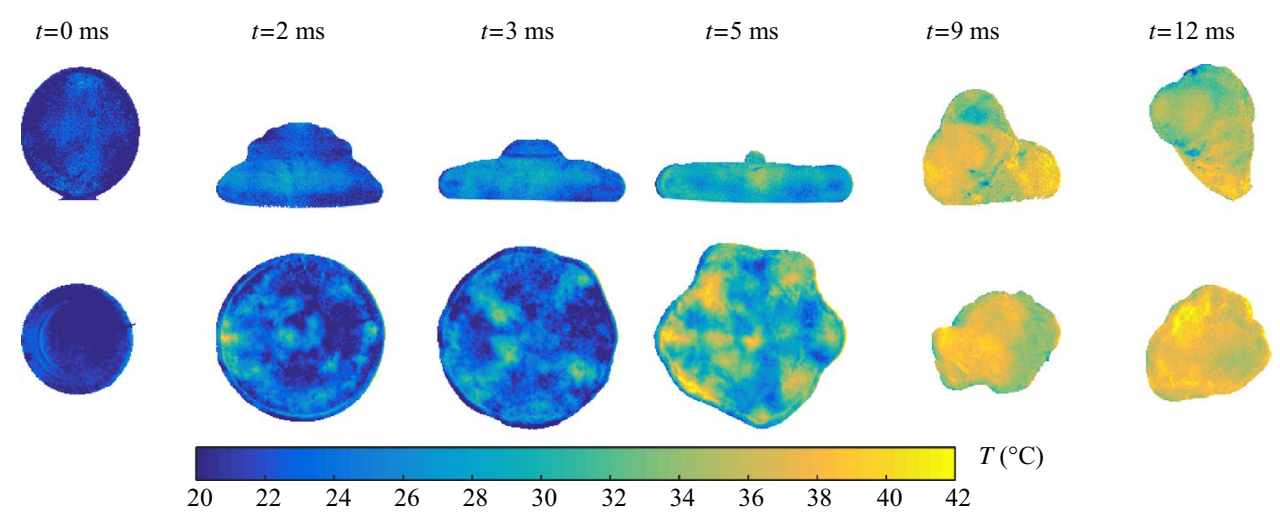

Fig. 11 Side and bottom views of the temperature field within an impacting droplet at $W e=30.6$

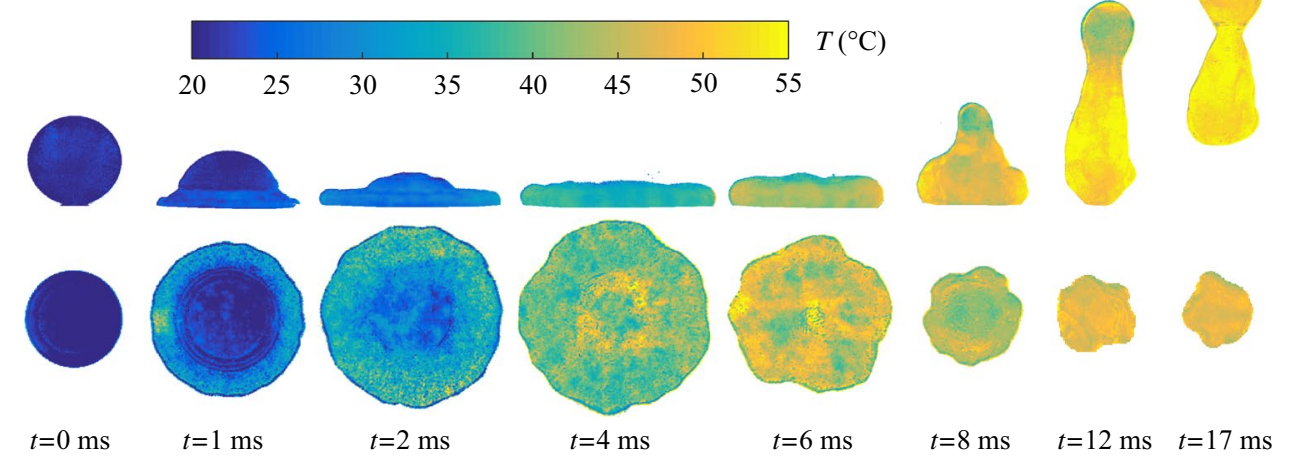


views of the temperature field within an impacting droplet at $W e=64.7$
Fig. 12 Side and bottom

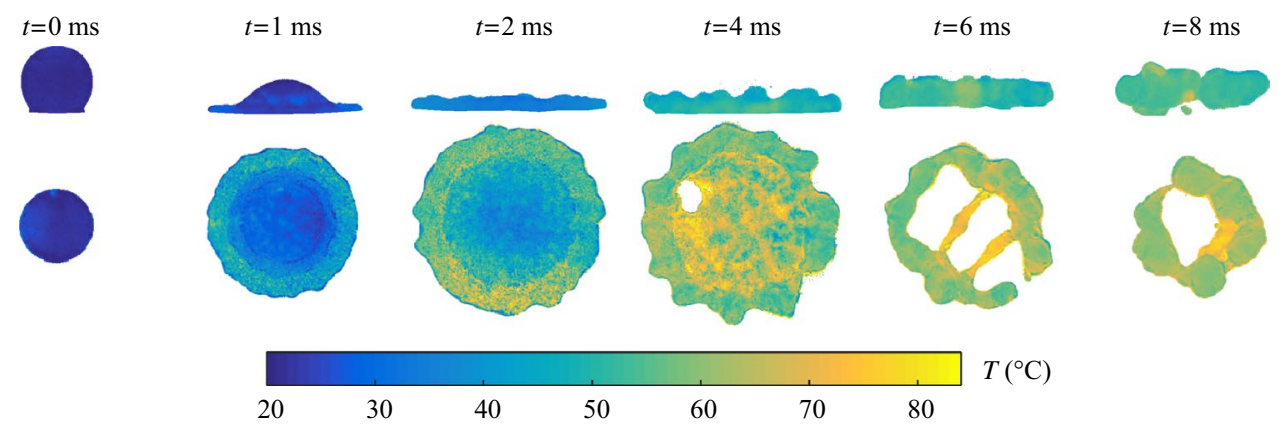

Fig. 13 Side and bottom views of the temperature field within an impacting droplet at $W e=92$

Fig. 14 Side and bottom views of the temperature field within an impacting droplet at $W e=140$

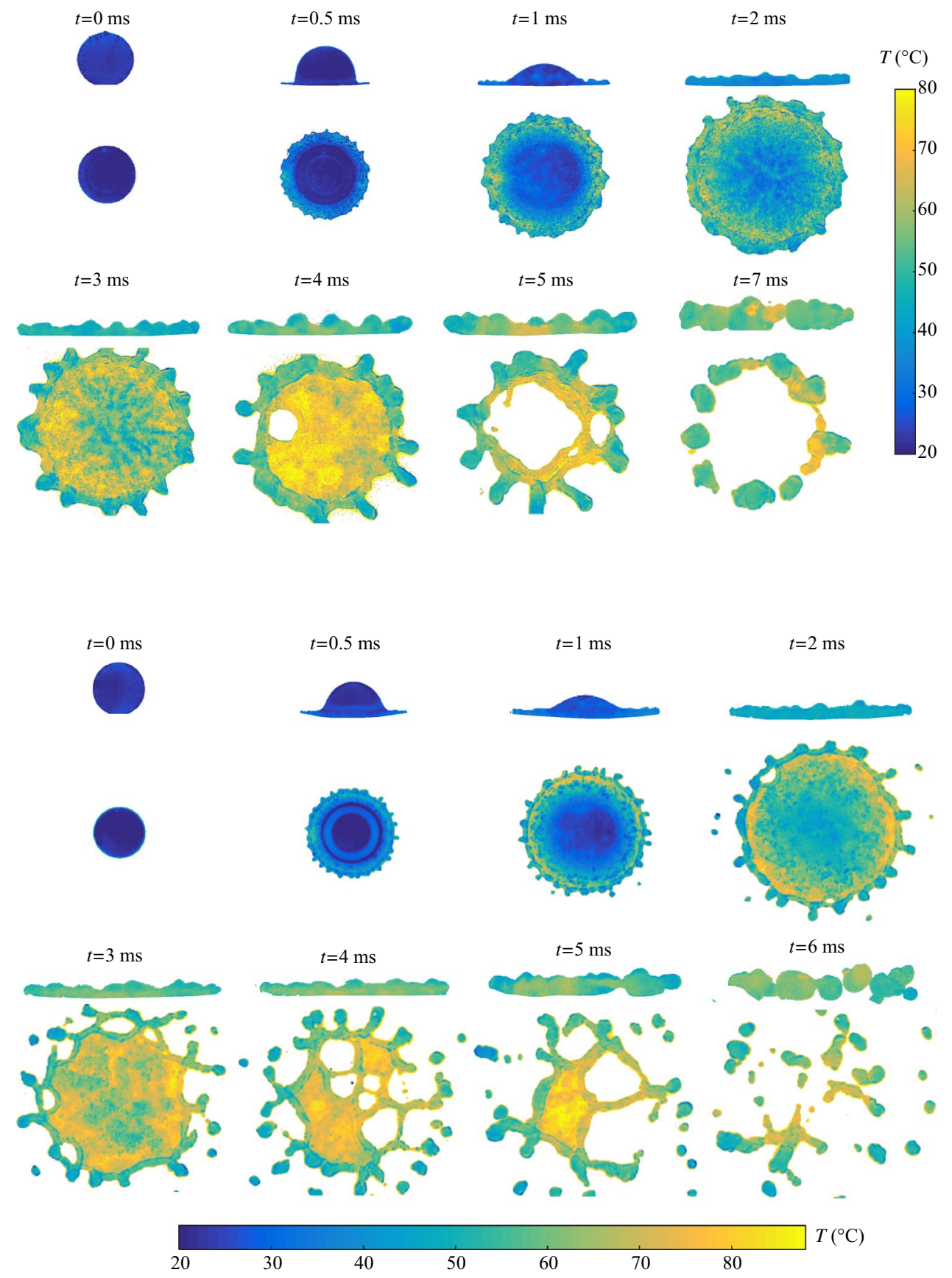




\section{Experimental results}

The experiments consist in varying the falling height of the droplet and thus its impact velocity $V$. Side and bottom views of the collision between the droplet and the wall are recorded for each impact condition. The Weber number $W e=\rho V^{2} d / \gamma$, which compares the kinetic energy of the droplet $\left(\sim \rho V^{2}\right)$ to its surface energy $(\sim \gamma / d)$, ranges from 10 to 140 . The magnification of the imaging system is adjusted for each experimental case to observe the whole droplet deformation, given that the maximum spreading diameter is increasing drastically with the Weber number. The wall temperature is set to $600{ }^{\circ} \mathrm{C}$, while the initial droplet temperature is maintained at $20{ }^{\circ} \mathrm{C}$. Figures 10 , $11,12,13,14$ show instantaneous images of the temperature taken at different times during the impact. To interpret these images, it should be kept in mind that

- The depth of field is several millimeters. The optical system is not able to provide an optical sectioning of the droplet in the same way as microscope tomography. Instead, the images roughly correspond to an average of the temperature in the depth of the droplet.

- Light refraction at the liquid interface is responsible for a distortion of the images. No correction is performed because the shape of the droplets is quite complex.

Lack of signal can be critical for single shot measurements of the temperature. For improving the signal-tonoise ratio, increasing the energy of the laser is not a solution because the fluorescence signal is already saturated. Increasing the concentration of the dyes beyond a certain limit is detrimental because of the energy transfers between the two dyes as explained in Sect. 3.2.2. Based on Fig. 8b, the dye concentration was fixed at $C_{\mathrm{SR} 640}=7 \cdot 10^{-7} \mathrm{M}$ and $C_{\mathrm{FL}}=2 \cdot 10^{-4} \mathrm{M}$. Ultimately pixel binning, i.e., the combination of charges from adjacent pixels of the CCD sensors, was performed to improve the signal-to-noise ratio albeit at the expense of reduced spatial resolution. Pixel binning is, respectively, $4 \times 4$ and $8 \times 8$ for side and bottom view images. Given the 8 megapixels of the cameras, the loss of spatial resolution due to pixel binning remains acceptable (approximately $10 \mu \mathrm{m}$ for the side view images and $20 \mu \mathrm{m}$ for the bottom view images).

In the following description, the spreading, the recoiling and the fragmentation of the droplet are considered separately.

Spreading The spreading phase lasts about 3-4 ms. Even if the bulk of the droplet maintains its initial temperature in the early stage of the spreading, there is necessarily a thin liquid layer close to the interface with the vapor film, where the liquid temperature reaches about $100{ }^{\circ} \mathrm{C}$. However, this liquid layer is too thin to be observed on the side view images. Its contribution to the bottom views is all the more important than the liquid thickness is small. In the case of $W e=10.2$, the heating is rather moderate during the spreading. In Fig. 10, it is difficult to point out a region where the heating is more intense. At higher Weber numbers (Figs. 11, 12, 13, 14), bottom views show a higher temperature band around the edge of the droplet at $t=1 \mathrm{~ms}$ and $2 \mathrm{~ms}$. At these times, the ejected lamella is much thinner than the central region of the droplet, which helps observing a heating of the liquid in the bottom views. Provided a sufficiently large impact velocity, the lamella rapidly takes a gaussian shape surrounded by an annular rim, which is growing due to the liquid deceleration induced by the capillary forces opposed to the spreading (Castanet et al. 2015). Liquid is progressively heated while flowing along the hot wall from the core of the lamella in the direction of the rim at the edge of the droplet. In Figs. 13 and 14, the liquid temperature apparently reaches almost $80^{\circ} \mathrm{C}$ in the region of minimum thickness just before the entrance of the rim. Afterwards, in the rim, the hot liquid coming from the lamella rapidly mixes with colder liquid already accumulated there. Disturbances on the rim of the spreading drop can be easily observed for $W e=92$ and $W e=140$ at the early stage of the impact. These disturbances increase with time, leading to a spatter with characteristic fingers. Disturbances of smaller amplitudes also develop for lower Weber numbers but much more slowly. Even for $W e=10.2$, the droplet does not keep a perfectly circular shape in the bottom views. It was proposed that fingering is caused by the Rayleigh-Taylor instability because a heavier fluid (water) is decelerated with respect to a lighter one (air) during the spreading (Thoroddsen and Sakakibara 1998; Mehdizadeh et al. 2004). In this view, the number of fingers and their growth rate are determined by the magnitude of the rim deceleration. At $t=4$ and $5 \mathrm{~ms}$, it can be seen for $W e=92$, both on the side and bottom view images, that the fingers are colder than the thinner regions between them in the rim.

Fragmentation of the rim and splashing For We $=92$ and $W e=140$, the previously mentioned fingers detach from the rest of the rim causing the fragmentation of the rim and ultimately the splashing of the droplet. The temperature of the secondary droplets resulting from the rim fragmentation evolves very little with time. It is of the order of $55-60{ }^{\circ} \mathrm{C}$ for both $W e=92$ and $W e=140$. In parallel, the lamella becomes so thin at the end of the spreading that it breaks. When this occurs, the temperature of the thin liquid sheet (about $80^{\circ} \mathrm{C}$ ) is much larger than that of the surrounding liquid in the rim. Holes usually open in the 
vicinity of the rim where the lamella is thinner. The holes rapidly expands according to the Taylor-Culick theory (Culick 1960). During this process, hot liquid ligaments are created because of the opening and expansion of several holes at the same time.

Recoiling and bouncing The recoiling of the droplet is initiated by the surface forces acting on the rim edge. The case $W e=64.7$ has some similarities with $W e=92$ and $W e=140$. Here also, several holes open the lamella at the end of the spreading phase. However, the rim does not break up and the empty space replacing the lamella narrows during the recoiling phase. For $W e=10.2$ and $W e=30.6$, the temperature is not uniform when the droplet takes off from the solid surface at about $t=12 \mathrm{~ms}$. For $W e=30.6$, the temperature is lower at the top end of the droplet where a satellite droplet nearly detaches. In Fig. 10, mixing currents are visible at $t=9 \mathrm{~ms}$ and $t=12 \mathrm{~ms}$, while the droplet is about to leave the wall. Hence, the flow induced by the droplet deformation inside of it is not capable of a full mixing of the liquid when the impact velocity is weak in the bouncing regime, typically for $W e \leq 30$.

Influence of the impact velocity Figure 15 shows the temporal evolution of the droplet heating for all the tested impact velocities. The reported values are calculated from the spatial averages of the side and bottom view images presented in Figs. 10, 11, 12, 13, 14. Whatever the Weber number, it is observed that the heating evaluated from the bottom view images is systematically larger than that determined from the side view images. Obviously, both temperatures converge to the same values at the end of the impingement. Median trend evolutions (i.e., the mean temperature between the

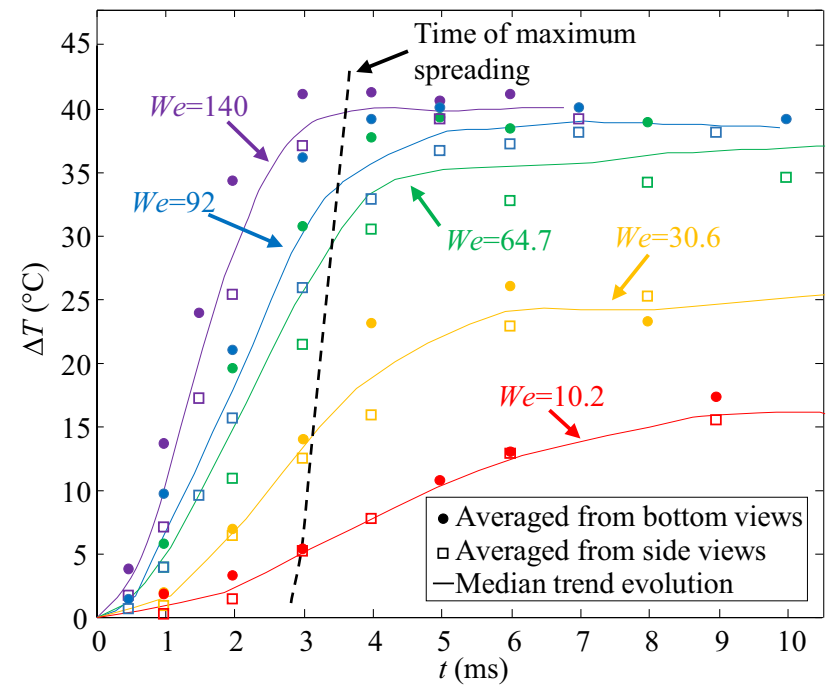

Fig. 15 Temporal evolution of the spatially averaged droplet heating bottom and side view images) are plotted as solid line curves in Fig. 15. The median is assumed to be closer to the true volume-averaged temperature of the droplet. In a first phase, the heating increases with increasing Weber number, then in a second phase a plateau is reached, meaning that We has almost not influence on the final liquid temperature in the splashing regime contrary to the bouncing regime. This trend has already been observed by Castanet et al. (2009) and Dunand et al. (2013), who also measured an heating of about $40{ }^{\circ} \mathrm{C}$ in the case of the splashing of micrometer size droplets with an initial temperature of $20^{\circ} \mathrm{C}$. The duration of the heating phase decreases with We. At low impact velocity, the heating period covers both the spreading and the recoiling phases. For $W e=10.2$, the heating is about one third of its final value at the end of the spreading. On the contrary, most of the heating takes place during the spreading for $W e \geq 64.7$. For higher Weber numbers, the heating has reached (or is about to reach) its final value just before that the fragmentation of the rim takes place. Hence, measurements reveal that the fragmentation of the droplet in itself has little effect on the final heating. During the spreading, the heat exchange with the wall is greatly enhanced by the creation of surface under the droplet. This seems to be the main reason for the differences observed among the experimental cases.

\section{Measurement uncertainties and biases}

\subsection{Measurement uncertainties}

The noise of the acquired images is responsible for uncertainties on the measured temperatures. The rms of the read noise slightly increases with the gray level of the pixels. It approximatively varies from 10 to 40 while the gray level increases from 300 to 4096 . In the same time, the dark current noise is limited to about 2 levels. Given that there is no simple way to assess the rms of the ratio between two randomly distributed variables, generators of random numbers are used to evaluate how errors due to the camera noise propagate to the fluorescence ratio and then to the temperature. The procedure has been described by Chaze et al. (2016). Denoting $I_{1}$ and $I_{2}$ the signal levels measured by the cameras, two series of about 100000 numbers that follows a normal distribution are created. The mean values of these series are, respectively, $I_{1}$ and $I_{2}$ while their rms are fixed with respect to the signal-to-noise characteristics of the cameras. For each couple of numbers present in the series, the fluorescence ratio and the temperature are calculated. The rms of the temperature $\sigma_{\mathrm{T}}$ is finally determined. Pixel binning is also accounted for. It makes 'super pixels' with a reduced read noise contributions, while the dark current noise not reduced by binning remains nearly negligible. Figure 16 shows an image of $\sigma_{\mathrm{T}}$ for two examples 


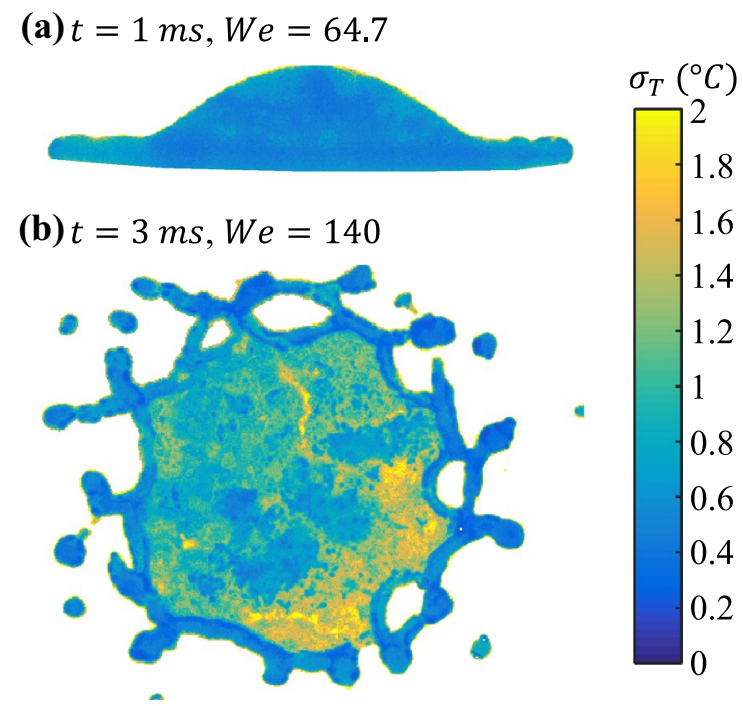

Fig. 16 Estimated uncertainties on the measured temperature for two experimental cases

corresponding to a side view and a bottom view. The thin liquid sheet at the center of the droplet in Fig. 16b is among the worst conditions for accurately measuring the temperature due to the limitation of the signal level. Despite this low signal, $\sigma_{\mathrm{T}}$ remains limited to about $2{ }^{\circ} \mathrm{C}$. In most of the situations, $\sigma_{\mathrm{T}}$ is lower than $1{ }^{\circ} \mathrm{C}$. Obviously, there are other sources of uncertainties in the measurements. For instance, errors on the reference temperature yield systematic errors on the measured temperature but this error should not affect the evaluations of the liquid heating. Errors on the temperature calibration would have an effect on all the measurements and even on the evaluations of the droplet heating. Much care must be taken to limit these errors to the maximum (high level signal, stirring of the liquid, etc.). In addition, the calibrations were repeated several times to confirm the values obtained for the coefficients in Eq. (5).

Obviously, there are also some uncertainties for the determination of the calibration curve. Based on several calibrations, it was estimated that the uncertainty $\Delta s$ on the temperature sensitivity of the fluorescence ratio is about $\pm 0.05 \% /{ }^{\circ} \mathrm{C}$. Based on Eqs. (2) and (4), an estimate of the temperature uncertainty $\Delta T$ can be easily obtained,

$\exp \left\{s \cdot\left(T_{\mathrm{m}}-T_{0}\right)\right\}=\exp \left\{(s+\Delta s) \cdot\left(T_{\mathrm{m}}+\Delta T-T_{0}\right)\right\}$,

where $T_{\mathrm{m}}$ is the measured temperature and $T_{0}$ is the reference temperature mentioned in Eq. (8). This expression yields

$\Delta T=\frac{\Delta s}{s+\Delta s}\left|T_{m}-T_{0}\right|$
In paragraph 3.2.2, it was asserted that the value of $s$ is about $2.7 \% /{ }^{\circ} \mathrm{C}$. Taking for $T_{\mathrm{m}}$ the value of $90{ }^{\circ} \mathrm{C}$ (i.e., the highest measured temperature in the experiments) and $20{ }^{\circ} \mathrm{C}$ for $T_{0}, \Delta T$ is equal to $\pm 1.2{ }^{\circ} \mathrm{C}$ according to Eq. (11). If $T_{\mathrm{m}}=75{ }^{\circ} \mathrm{C}$ and $T_{0}=20{ }^{\circ} \mathrm{C}, \Delta T= \pm 1{ }^{\circ} \mathrm{C}$. Hence, the measurement error due to the calibration is expected to be less than $1{ }^{\circ} \mathrm{C}$ for most of the measurements.

\subsection{Repeatability of the drop impacts}

The droplet generation is a perfectly repeatable process (less than $0.3 \%$ variation in size between the droplets). The optical barrier has an electronic jitter of $10 \mu$ $\mathrm{s}$, which is very small in comparison with the duration of the impact process (about $10 \mathrm{~ms}$ ). When a droplet impinges onto a sapphire wall, the temperature at the sapphire surface decreases temporarily. The modelling of the heat conduction inside the sapphire windows shows that the upper surface of sapphire recovers its initial temperature (namely $600{ }^{\circ} \mathrm{C}$ ) in less than $1 \mathrm{~s}$. Droplets being generated every $10-30 \mathrm{~s}$, there is no risk that the wall temperature could be affected by the thermal footprint of a previous droplet.

Nevertheless, differences between consecutive droplets can be observed especially in the recoiling phase at high Weber numbers, because surface instabilities do not always develop exactly in the same manner (orientation, amplitude of the deformations). When the lamella is becoming very thin and breaks, the number and the location of the holes are somehow random, although it was observed that the holes usually appears near the edge of the lamella. This is illustrated in Fig. 17 in the case of $W e=92$ and $W e=140$. Temperature images of three droplets impinging consecutively on the solid wall are presented for different impact times. The temperature distributions of consecutive droplets retain a certain degree of similarity, even if the droplet shape and the presence of holes and secondary droplets are changing from one drop to another.

\subsection{Measurement biases}

\subsubsection{Morphology dependence resonances and lasing of the fluorescent dyes}

Some of the fluorescence emitted by SR640 can be trapped inside morphology-dependent resonant modes (MDRs). Rays of fluorescence light turn around the surface of the droplet through successive internal reflections, and can be amplified because of constructive interference to the extent of giving rise to a stimulated emission of SR640. This effect is not observed in the case of FL because absorption by SR640 prevents any 
Fig. 17 Examples of temperature fields measured for three droplets impinging consecutively onto the solid wall. a $W e=92, \mathbf{b} W e=140$
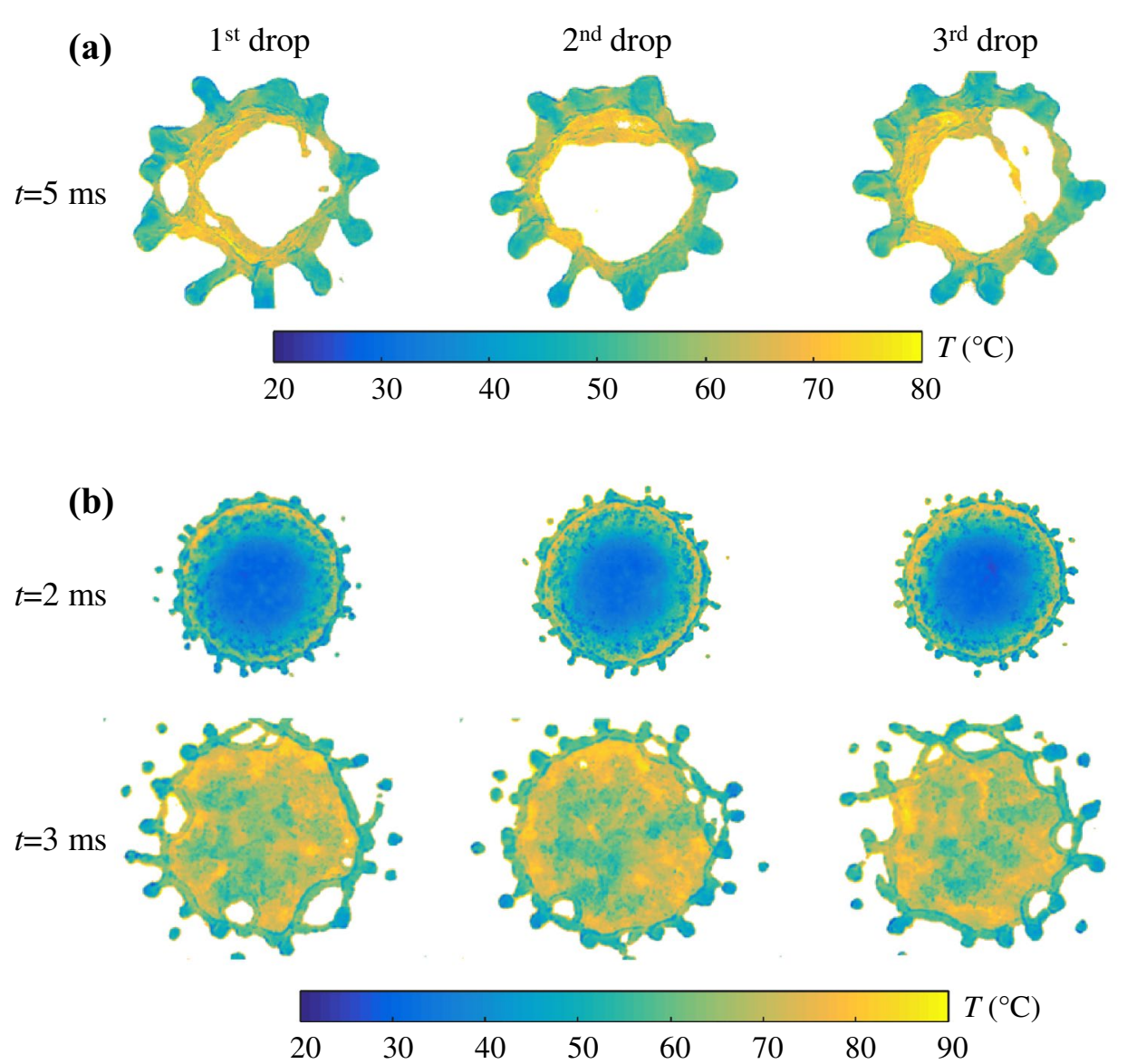

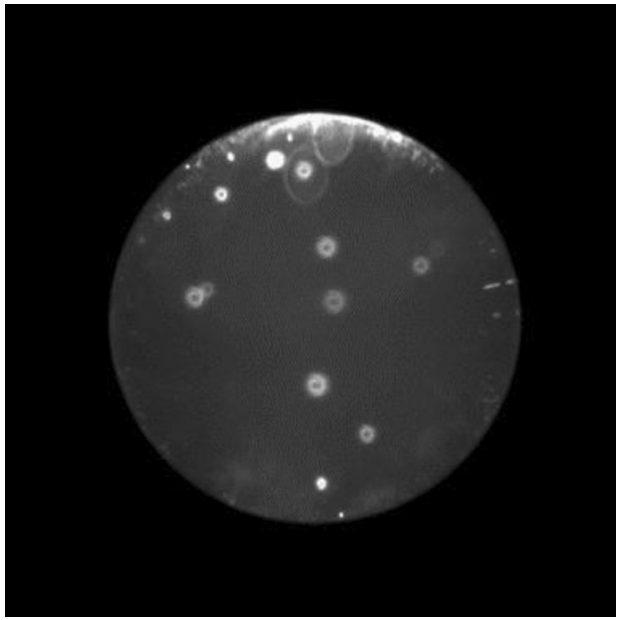

Fig. 18 Image illustrating the lasing emission of SR640 in the detection band $\lambda>640 \mathrm{~nm}$ in the case of a spherical free falling droplet. The concentration of SR640 has been increased to $3 \cdot 10^{-6} \mathrm{M}$ to enhance stimulated emission of SR640 and improve its visualization in the images without any pixel binning

amplification of FL emission in the MDRs. As a consequence of the MDRs, shiny spots are visible in the images detected in the band $\lambda>640 \mathrm{~nm}$ at the beginning

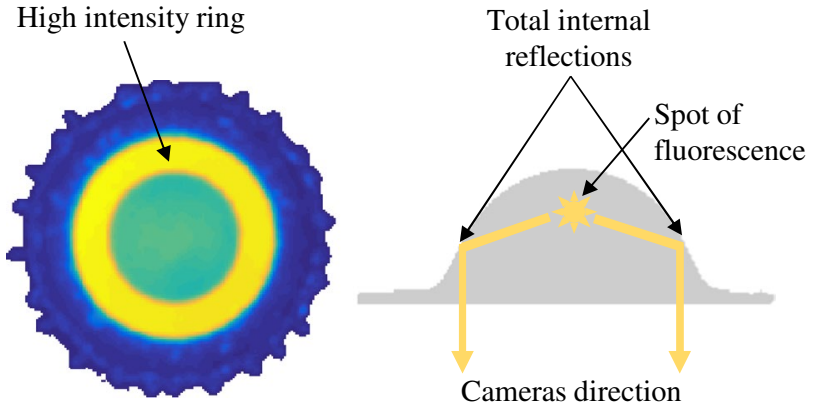

Fig. 19 Illustration of the measurement bias due to total internal reflections in the case of a bottom side view $(t=0.5 \mathrm{~ms}$ and $W e=140$, temperature map presented in Fig. 14)

of the impingement when the droplet still has a spherical shape (Fig. 18). Similar observations were reported for other fluorescence dyes and the effect on temperature measurement using the 2cLIF technique was discussed by Perrin et al. (2015). Presently, the effect of the MDRs is a bias of the fluorescence ratio at the very beginning of the drop impingement (i.e., at $t=0 \mathrm{~ms}$ in Figs. 10, $11,12,13,14)$. As the droplet loses its sphericity during the impact, the problem disappears. 


\subsubsection{Total internal reflections}

During its impingement, the droplet takes various shapes. For some of these shapes, a substantial part of the fluorescence can emerge from the droplet after having undergone one or several total internal reflections. This is illustrated in Fig. 19 for a drop configuration corresponding to $t=0.5$ $\mathrm{ms}$ and $W e=140$ (Fig. 14). A significant part of the fluorescence emitted in the top region of the droplet is totally reflected on the edge of the lamella. In doing so, rays of fluorescent light are deviated in the direction of the cameras observing the droplet from below. In the fluorescence images, a ring of high signal intensity is visible in the areas where totally reflected rays emerge from the droplet (Fig. 19). Because there are differences in temperature between the top region and the edge of the droplet, the effect of internal reflections is noticeable on the temperature image. A colder ring can be seen in the temperature map corresponding to $t=0.5 \mathrm{~ms}$ and $W e=140$ which is displayed in Fig. 14. Similar effects can be present on both side and bottom view images depending on the droplet shape.

\section{Conclusion}

Two-color laser-induced fluorescence thermometry has been implemented to obtain single-shot images of the temperature inside droplets impinging on a hot solid surface in the conditions of film boiling. The characterization of the transient temperature evolution is an interesting step forward for the modelling of the heat transfers between a drop and a hot solid surface. The use of a transparent sapphire window allows obtaining bottom and side view images of the temperature field, which are complementary to provide some insights into the heat transfers. Measurements show that the impact velocity modifies the thermal mixing and the rate of heat transfers. At high Weber numbers, corresponding to the splashing of the impacting droplet, the heating is almost completed at the end of the spreading. The protrusions, formed by the instability of the rim at the edge of the droplet, are significantly colder than the rest of the liquid (lamella, ligaments). The secondary droplets resulting from the fragmentation of the rim leave the solid surface with almost the same temperature, which depends very little on the impact velocity. In contrast, the heating of bouncing droplets sharply increases with the Weber number and takes place over the entire deformation process, i.e., both the spreading and recoiling phases. After its rebound, the droplet leaves the solid surface with a non-homogeneous temperature distribution, the top end of the droplet being colder. These first investigations were limited to the role played by the impact velocity. The effects of parameters, such as the degree of subcooling of the incident droplet, the temperature of the solid surface, need to be quantified, as one can suspect an influence on the thickness of the vapor layer, and thus on the heat and mass transfers.

This study also demonstrates the capabilities of the twocolor laser-induced fluorescence thermography to perform an accurate instantaneous temperature imaging of dispersed liquid flows. The couple of dyes fluorescein disodium/ sulforhodamine 640 should be recommended at high laser irradiances in the saturated regime of excitation of the fluorescence. Using two fluorescent dyes provide some benefits in comparison with the one-dye approach, also broadly described in the literature. It allows a substantial gain in temperature sensitivity and makes it possible to balance the signal in the two detection bands by adjusting the concentrations of the dyes. Nevertheless, the difficulties of the method should not be underestimated:

(i) Spectral conflicts, e.g., the emission band of the dye interfering with the emission of the second, must be anticipated and limited as much as possible because they undermine the sensitivity of the measurements.

(ii) In case of spectral conflicts, changing the dye concentration can affect the temperature sensitivity. Thus, serious problems can be encountered if one of the dyes is more affected than the other by photo- or thermal degradations.

(iii) Energy transfers between the two fluorescent species can be very detrimental to the measurements. A secondary fluorescence can be induced by the absorption of the fluorescent emission of one dye by the other. Radiationless transfers, such as Förster resonance energy transfers arising from dipole-dipole coupling, can be predominant, when the concentrations of the dyes are relatively high.

(iv) Droplet measurements are inherently biased by some artifacts such as morphological dependent resonances or total internal reflections at liquid-gas interfaces.

\section{References}

Bernardin JD, Mudawar I (1997) Film boiling heat transfer of droplet streams and sprays. Int J Heat Mass Transf 40(11):2579-2593

Bruchhausen M, Guillard F, Lemoine F (2005) Instantaneous measurement of two-dimensional temperature distributions by means of two-color planar laser induced fluorescence (plif). Exp Fluids 38(1):123-131

Castanet G, Lienart T, Lemoine F (2009) Dynamics and temperature of droplets impacting onto a heated wall. Int J Heat Mass Transf 52(3-4):670-679

Castanet G, Labergue A, Lemoine F (2011) Internal temperature distributions of interacting and vaporizing droplets. Int J Therm Sci 50(7):1181-1190 
Castanet G, Caballina O, Lemoine F (2015) Drop spreading at the impact in the Leidenfrost boiling. Phys Fluids 27(6):063302

Chaze W, Caballina O, Castanet G, Lemoine F (2016) The saturation of the fluorescence and its consequences for laser-induced fluorescence thermometry in liquid flows. Exp Fluids 57(4):58

Coppeta J, Rogers C (1998) Dual emission laser induced fluorescence for direct planar scalar behavior measurements. Exp Fluids 25(1):1-15

Crimaldi J (2008) Planar laser induced fluorescence in aqueous flows. Exp Fluids 44(6):851-863

Culick FEC (1960) Comments on a ruptured soap film. J Appl Phys 31(6):1128-1129

Dobrovinskaya ER, Lytvynov LA, Pishchik V (2009) Properties of sapphire. Springer, Boston

Dunand P, Castanet G, Lemoine F (2012) A two-color planar LIF technique to map the temperature of droplets impinging onto a heated wall. Exp Fluids 52(4):843-856

Dunand P, Castanet G, Gradeck M, Maillet D, Lemoine F (2013) Energy balance of droplets impinging onto a wall heated above the Leidenfrost temperature. Int J Heat Fluid Flow 44:170-180

Eggers J, Fontelos MA, Josserand C, Zaleski S (2010) Drop dynamics after impact on a solid wall: theory and simulations. Phys Fluids 22(6):062101

Jung J, Jeong S, Kim H (2016) Investigation of single-droplet/wall collision heat transfer characteristics using infrared thermometry. Int J Heat Mass Transf 92:774-783

Kim H, Kihm K, Allen J (2003) Examination of ratiometric laser induced fluorescence thermometry for microscale spatial measurement resolution. Int J Heat Mass Transf 46(21):3967-3974

Labergue A, Delconte A, Lemoine F (2013) Study of the thermal mixing between two non-isothermal sprays using combined threecolor lif thermometry and phase doppler analyzer. Exp Fluids 54(6): 1527

Labergue A, Gradeck M, Lemoine F (2015) Comparative study of the cooling of a hot temperature surface using sprays and liquid jets. Int J Heat Mass Transf 81:889-900

Labergue A, Pena-Carillo JD, Gradeck M, Lemoine F (2017) Combined three-color lif-pda measurements and infrared thermography applied to the study of the spray impingement on a heated surface above the Leidenfrost regime. Int $\mathbf{J}$ Heat Mass Transf 104:1008-1021

Lakowicz JR (2006) Principles of fluorescence spectroscopy. Springer, New York

Lemoine F, Castanet G (2013) Temperature and chemical composition of droplets by optical measurement techniques: a state-ofthe-art review. Exp Fluids 54(7):1572
Lemoine F, Antoine Y, Wolff M, Lebouche M (1999) Simultaneous temperature and $2 \mathrm{~d}$ velocity measurements in a turbulent heated jet using combined laser-induced fluorescence and lda. Exp Fluids 26(4):315-323

Liang G, Mudawar I (2017) Review of drop impact on heated walls. Int J Heat Mass Transf 106:103-126

Mehdizadeh NZ, Chandra S, Mostaghimi J (2004) Formation of fingers around the edges of a drop hitting a metal plate with high velocity. J Fluid Mech 510:353-373

Moreira A, Moita A, Panão M (2010) Advances and challenges in explaining fuel spray impingement: how much of single droplet impact research is useful? Prog Energy Combust Sci 36(5):554-580

Perrin L, Castanet G, Lemoine F (2015) Characterization of the evaporation of interacting droplets using combined optical techniques. Exp Fluids 56(2):29

Ranz W, Marshall W (1952) Evaporation from drops. Chem Eng Prog 48(141-146):173-180

Roisman IV, Berberović E, Tropea C (2009) Inertia dominated drop collisions. I. On the universal flow in the lamella. Phys Fluids 21(5):052103

Rueda Villegas L, Tanguy S, Castanet G, Caballina O, Lemoine F (2017) Direct numerical simulation of the impact of a droplet onto a hot surface above the Leidenfrost temperature. Int J Heat Mass Transf 104:1090-1109

Saha J, Roy AD, Dey D, Chakraborty S, Bhattacharjee D, Paul P, Hussain SA (2015) Investigation of fluorescence resonance energy transfer between fluorescein and rhodamine $6 \mathrm{~g}$. Spectrochim Acta A 149:143-149

Sakakibara J, Adrian R (1999) Whole field measurement of temperature in water using two-color laser induced fluorescence. Exp Fluids 26(1-2):7-15

Sakakibara J, Adrian R (2004) Measurement of temperature field of a Rayleigh-Benard convection using two-color laser-induced fluorescence. Exp Fluids 37(3):331-340

Sakakibara J, Hishida K, Maeda M (1993) Measurements of thermally stratified pipe-flow using image-processing techniques. Exp Fluids 16(2):82-96

Sutton JA, Fisher BT, Fleming JW (2008) A laser-induced fluorescence measurement for aqueous fluid flows with improved temperature sensitivity. Exp Fluids 45(5):869-881

Thoroddsen ST, Sakakibara J (1998) Evolution of the fingering pattern of an impacting drop. Phys Fluids 10(6):1359-1374

Walker D (1987) A fluorescence technique for measurement of concentration in mixing liquids. J Phys E-Sci Instrum 20(2):217-224 